\title{
Using transperineal ultrasound to predict labor onset
}

\author{
Fanghua Peng", Yang Yu\#, Yanan Sun, Shan Jiang, Yun Han, Zhikun Zhang \\ Pelvic Floor Research Group, Department of Ultrasound and Tianjin Key Laboratory of Human Development and Reproductive Regulation, \\ Tianjin Central Hospital of Gynecology Obstetrics, Tianjin 300100, China \\ Contributions: (I) Conception and design: F Peng, Z Zhang, Y Yu; (II) Administrative support: None; (III) Provision of study materials or patients: \\ Y Yu; (IV) Collection and assembly of data: Y Yu; (V) Data analysis and interpretation: Y Sun, Y Han, J Shan, Y Yu; (VI) Manuscript writing: All \\ authors; (VII) Final approval of manuscript: All authors. \\ \#These authors contributed equally to the work. \\ Correspondence to: Zhikun Zhang. Department of Ultrasound and Tianjin Key Laboratory of Human Development and Reproductive Regulation, \\ Tianjin Central Hospital of Gynecology Obstetrics, No. 156 Nankai Sanma Road Nankai District, Tianjin 300100, China. Email: zkunlin@sina.com.
}

Background: Accurate diagnosis of labor onset is especially important for the counseling about elective induction of labor. The study aimed to evaluate whether transperineal ultrasound could better predict the timing and likelihood of spontaneous labor at term.

Methods: This single-center study was conducted between 2018 and 2019.Data on singleton pregnant women after 39 weeks and before labor onset were retrospectively reviewed.

Results: All the transperineal ultrasound parameters were well reproducible between the two doctors [intraclass correlation coefficient (ICC) for progression distance(PD) was $0.892, \mathrm{P}<0.001$ ], [ICC for angle of progression (AoP) was 0.881, $\mathrm{P}<0.001$ ], [ICC for subpubic arch angle (SPA) was $0.766, \mathrm{P}<0.001$ ], [ICC for width of symphysis pubis (WSP) was $0.803, \mathrm{P}<0.001]$. For the pregnant women before 40 weeks, the width of symphysis pubis changed gradually with the of spontaneous labor (WSP) $(\mathrm{r}=0.33, \mathrm{P}<0.05)$. For all included women, the SPA correlated with the time of spontaneous labor (SPA) $(r=0.31, \mathrm{P}<0.05)$.

Conclusions: The antepartum transperineal ultrasound is a simple and objective technique that better observes the initiation of labor. And with the WSP and SPA we were able to predict labor onset and help in counseling about elective induction of labor.

Keywords: Labor onset; progression distance (PD); angle of progression (AoP); subpubic arch angle (SPA); width of symphysis pubis (WSP)

Submitted Nov 11, 2018. Accepted for publication Nov 26, 2019.

doi: $10.21037 /$ atm.2019.12.11

View this article at: http://dx.doi.org/10.21037/atm.2019.12.11

\section{Introduction}

Proving the onset of labor is currently reliant on digital evaluation and a series of medical manifestations. Studies, however, have shown that digital analysis was poorly reproducible and inaccurate measurements were produced $(1,2)$ A key point in determining whether to induce labor is when is the start of labor. By avoiding the cost of increased use of resources in women who are likely to spontaneously undergo labor within a relatively short time, an accurate predictive model of labor onset is very useful for elective labor induction.
Transperineal ultrasound has been used to assess fetal head passing through the birth canal in labor. It is a potential way to improve the clinical practice of evaluation of the delivery process (3-5). Based on previous studies, progression distance $(\mathrm{PD})$ and angle of progression (AoP) were proven to be credible values to assess the fetal station (6).

At present, the measurement of pelvic diameter relies on radiographic examination, such as X-ray, CT, which cannot be routinely used for pelvic measurement of pregnant women. The results of the present researches suggested that ultrasound could be used to measure the subpubic arch angle (SPA) and the width of the symphysis pubis (WSP), 
Page 2 of 7

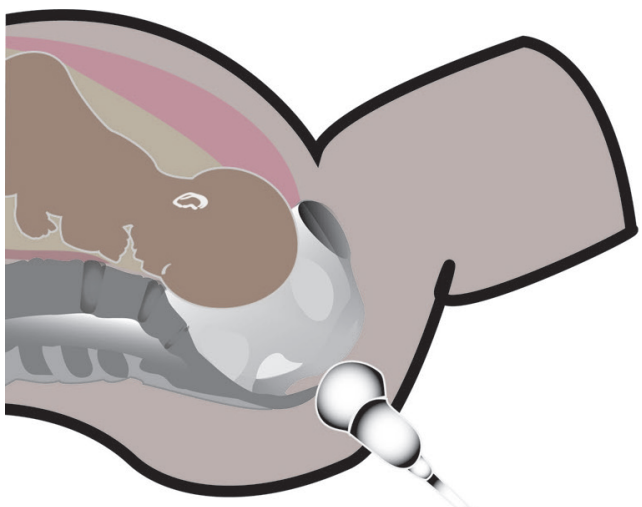

Figure 1 The ultrasound transducer is placed on the perineum in a mid-sagittal position, between the labia, and below the pubic symphysis.

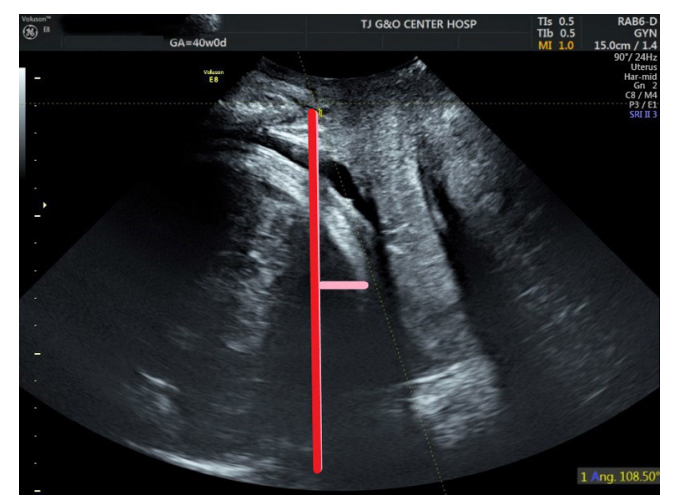

Figure 2 Transperineal ultrasound image (sagittal view) PD (pink line) is the distance between the lower edge symphysis pubis and the closest point of the fetal skull along a line passing perpendicular to the longitudinal axis of the symphysis pubis and tangential to its lower edge. PD, progression distance.

which can evaluate the bony birth canal $(7,8)$.

As we know, the labor process is a continuous process of fetal head descent, so we hypothesize that the above ultrasound parameters can also be applied to prenatal ultrasound to predict labor onset. This study aimed to evaluate fetal head descent and assess the correlation between ultrasonic parameters (PD, AoP, SPA, the WSP) and labor onset from two aspects of the fetal head station and birth canal.

\section{Methods}

This single central cross-sectional study only involved singleton pregnancies who given birth after 39 weeks of

\section{Peng et al. Transperineal ultrasound can predict labor onset}

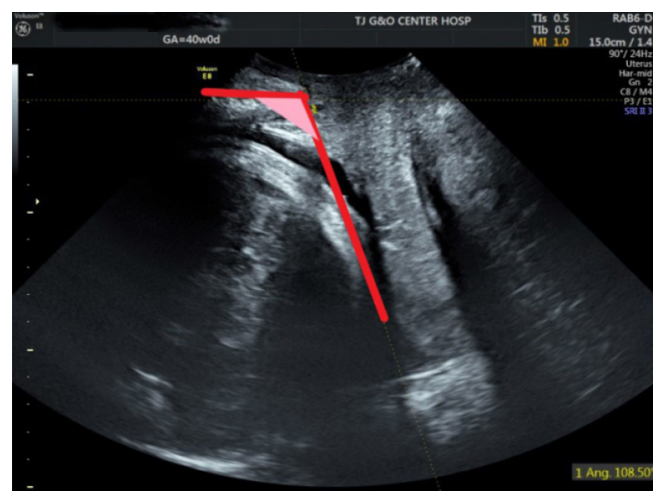

Figure 3 Transperineal ultrasound image (sagittal view) AoP (pink triangle) is the angle between the long axis of the pubic symphysis and the line extending from the most inferior point of the symphysis tangentially to the fetal skull contour (the two red lines). AoP, angle of progression.

gestation from November 5, 2018, and March 31, 2019, in Tianjin Central Gynecology Obstetrics Hospital. The local ethics committee approved the study protocol. Eligible patients received oral and written information and provided signed consent forms. Pregnancies met the following inclusion criteria: (I) singleton pregnancies, (II) no medical and surgical disease, (III) cephalic presentation, (IV) older than 18 years of age, (V) no premature rupture of membranes, (VI) no previous cesarean section.

Transperineal ultrasound measurements were performed using published methods with a $6-\mathrm{MHz}$ transabdominal probe (GE VolusonE8, General Electric, China). Four-dimensional (4D) ultrasound volumes and twodimensional (2D) ultrasound were acquired transperineally in the midsagittal plane as previously described. All measurements were performed by two operators with experience in $4 \mathrm{D}$ ultrasound. The measurements of $\mathrm{PD}$ and AoP were obtained using the symphysis pubis as reference points (9). The PD was defined as the distance between the lower edge symphysis pubis and the closest point of the fetal skull along a line passing perpendicular to the longitudinal axis of the symphysis pubis and tangential to its lower edge (6) (Figures 1,2). The AoP was stated as the angle between the long axis of the pubic bone and a line joining the lowest edge of the pubis to the lowest convexity of the fetal skull (Figure 3) $(8,10)$. After the $2 \mathrm{D}$ measurements, the volumes of SPA and WSP were acquired in the same central plane of discontinuity of pelvis, and the operators measured immediately following its acquisition (Figures 4-6). 


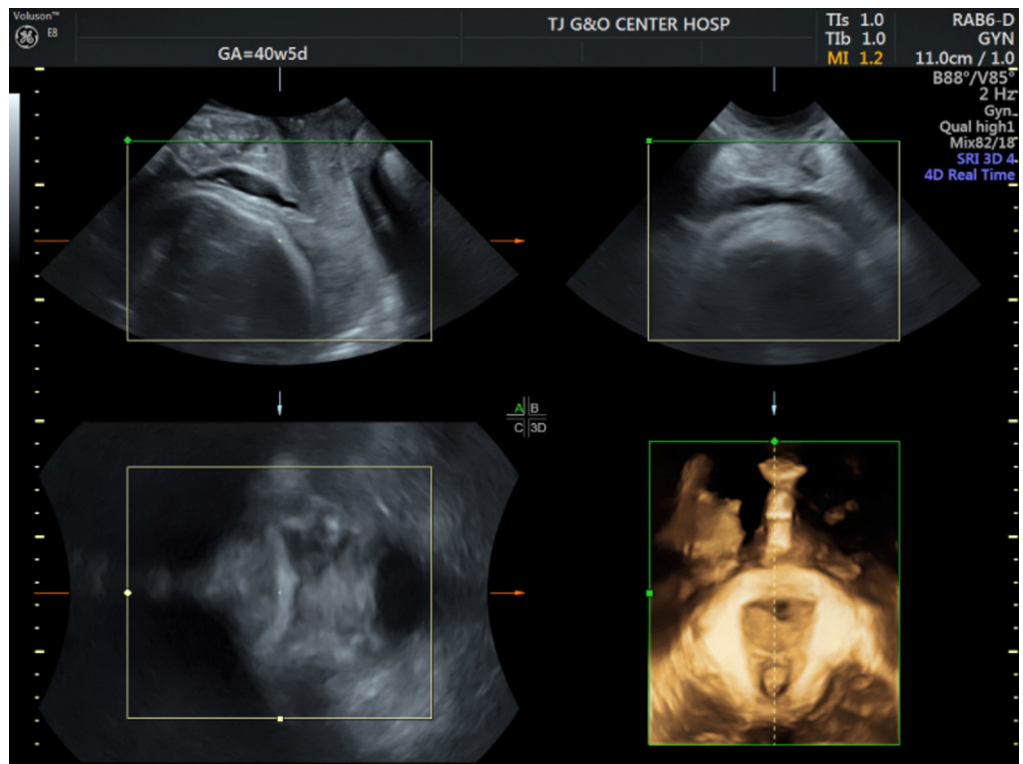

Figure 4 4D views of symphysis pubis joint and measurement of the wide symphysis pubis (the pink line). 4D, four-dimensional.

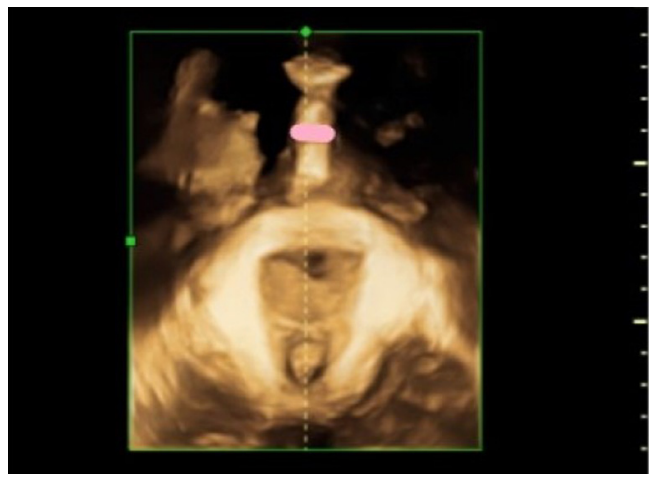

Figure $54 \mathrm{D}$ volumes were acquired using the transperineal ultrasound. 4D, four-dimensional.

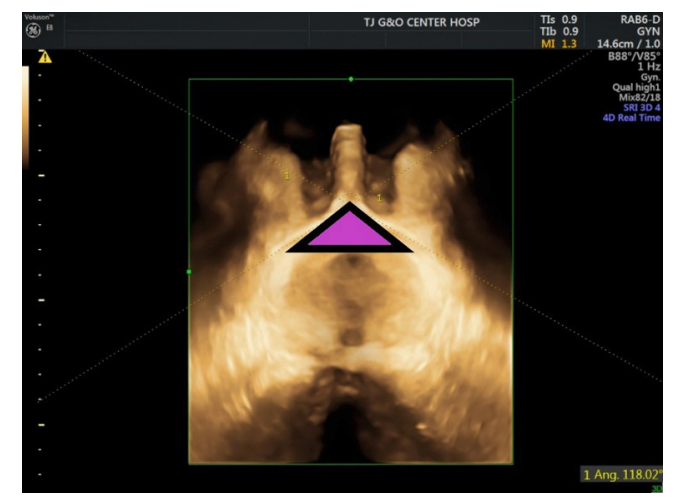

Figure 6 The plane of the pubic rami is then automatically displayed. The 3-point or the 2-line functions then measure the angle formed by the 2 pubic rami.
Women were excluded if they had a scheduled cesarean delivery without spontaneous labor. Women were included spontaneous labor and cesarean delivery with spontaneous. It is accepted that the gestation period of a normal pregnant woman is 40 weeks or 280 days. The length of the menstrual cycle determines the time of labor, which is more than 40 weeks when the menstrual cycle is long. Therefore, this study took 40 weeks as the point to conduct subgroup analysis on the data $(11,12)$. So, the pregnancies were divided into two subgroups depending on the gestation (at 39-40, 40+ weeks). All patients included in the study group, the time of labor onset, were retrospectively assessed. The correlation between labor onset and the measurement of PD, AoP, SPA, and WSP was investigated.

Mean, standard deviation (SD) were used as descriptive values. Multivariable logistic regression analyses were performed by taking into possible confounders, which included maternal age, body weight, height, gestational age at delivery, and parity, to identify their role in the prediction of labor onset. Linear regression analysis was conducted to estimate the association between ultrasound measurements and labor onset. Pearson correlation coefficient $(r)$ was used to evaluate the correlations. The intraclass correlation coefficient (ICC) was used to examine the intraobserver variability. Data were analyzed by using the SPSS (version 21.0), and $\mathrm{P} \leq 0.05$ was considered statistically significant. 
Table 1 Comparison of maternal and obstetrical characteristics between groups

\begin{tabular}{lcccc}
\hline Basic information & $40+$ weeks $(\mathrm{N}=23)$ & $39-40$ weeks $(\mathrm{N}=38)$ & Summation $(\mathrm{N}=61)$ & $\mathrm{P}$ \\
\hline Age (years) & $28.13 \pm 3.17$ & $30.16 \pm 4.48$ & $29.29 \pm 4.12$ & 0.062 \\
Height $(\mathrm{cm})$ & $165.35 \pm 5.23$ & $163.71 \pm 5.02$ & $164.33 \pm 5.12$ & 0.229 \\
Weight $(\mathrm{kg})$ & $74.07 \pm 9.60$ & $74.23 \pm 9.51$ & $74.17 \pm 9.47$ & 0.949 \\
\hline
\end{tabular}

Data are presented as the mean \pm standard deviation (SD). Age, gestational age, weight, height.

Table 2 ICC for intraobserver variability

\begin{tabular}{lllll}
\hline Variable & $\mathrm{N}$ & ICC & $95 \% \mathrm{Cl}$ & $\mathrm{P}$ value \\
\hline PD & 60 & 0.892 & $0.826-0.934$ & $<0.001$ \\
AoP & 60 & 0.881 & $0.809-0.927$ & $<0.001$ \\
WSP & 60 & 0.803 & $0.692-0.877$ & $<0.001$ \\
SPA & 60 & 0.766 & $0.639-0.853$ & $<0.001$ \\
\hline
\end{tabular}

Data are presented as the mean \pm standard deviation (SD). ICC, intraclass correlation coefficient; PD, progression distance; AoP, angle of progression; SPA, subpubic arch angle; WSP, width of symphysis pubis. $\mathrm{P}<0.05$ is statistics significance.

\section{Results}

Between November 5, 2018, and March 31, 2019, 67 eligible women participated in the study, and all of them consented to participate in this study. Among the women, $53(79.1 \%)$ had a spontaneous vaginal delivery, and 10 $(16.4 \%)$ underwent operative delivery (8 cesareans after labor onset and 2 cesareans deliveries without labor onset), $4(4.5 \%)$ lost to follow-up. There were no statistically significant differences in age, height, weight (Table 1).

Reproducibility of intrapartum transperineal ultrasound was good, ICC for intraobserver variability was 0.892 for $\mathrm{PD}(\mathrm{P}<0.001)$, and was 0.881 for $\mathrm{AoP}(\mathrm{P}<0.001)$, was 0.766 for SPA $(\mathrm{P}<0.001)$, was 0.803 for WSP $(\mathrm{P}<0.001)$ (Table 2$)$.

For all participants, the logistic regression showed that SPA was significantly related to labor onset $(r=0.31$, $\mathrm{P}<0.05)$. The closer to labor, the greater SPA is. There was no correlation between other ultrasonic parameters and labor onset (PD r=0.23, $\mathrm{P}>0.05$, AoP $\mathrm{r}=0.01, \mathrm{P}>0.05$, WSP $\mathrm{r}=0.20, \mathrm{P}>0.05)$ (Table 3, Figure 7).

For 39-40 weeks pregnant women, the results showed that WSP is significantly associated with labor onset $(r=0.33$, $\mathrm{P}<0.05)$. WSP is much bigger when the pregnant women are due to labor. There was no association of other ultrasonic measurements and labor onset ( $\mathrm{PD} \mathrm{r}=0.19, \mathrm{P}>0.05$, AoP $\mathrm{r}=0.04, \mathrm{P}>0.05$, SPA r=0.32, $\mathrm{P}=0.05$ ) (Table 4, Figure 8).

For 40+ weeks pregnant women, the results showed that PD, AoP, SPA and WSP did not correlate with labor onset $(\mathrm{PD} r=0.35, \mathrm{P}>0.05$, AoP $\mathrm{r}=0.09, \mathrm{P}>0.05$, SPA $\mathrm{r}=0.13$, $\mathrm{P}>0.05$, WSP $\mathrm{r}=-0.15, \mathrm{P}>0.05$ ) (Table 5).

However, there was no association between PD, AoP, and labor onset. We also observed there was probably cephalopelvic disproportion when the station of the head is high. Additionally, fetuses in the occiput posterior position are a long time away from labor onset.

\section{Discussion}

Most of our current knowledge about WSP and SPA for pregnancies was derived from either X-ray or MRI studies. A recent study found that WSP and SPA were wider in late pregnancy than nonpregnancy. In late pregnancy, WSP is less than $15 \mathrm{~mm}$, and the SPA is between $76^{\circ}$ to $110.6^{\circ}$. In the nonpregnancy, WSP is between 2.6 to $10.1 \mathrm{~mm}$, and SPA is more than $90^{\circ}(7,13,14)$. Using ultrasound, we achieved a similar result to the previous studies. In the previous papers, the reliability of the X-ray and ultrasonic measurement of the pubic symphysis is compared, and the result of the ultrasonic measurement is reliably (15). All these changes can be tied to the release of the hormone relaxin. At the tenth to twelfth week of pregnancy, the corpus luteum and placenta secrete the relaxin, which could increase the pelvic laxity and the WSP and SPA (16). The increasing WSP and SPA can enlarge the dimension of the pelvic entrance plane, ischial spinous plane, and pelvic outlet plane, and all of these changes could increase the 
Table 3 For all included pregnancies the association with labor onset

\begin{tabular}{lcc}
\hline Variable & Pearson correlation coefficient & $P$ \\
\hline PD $(\mathrm{mm})$ & 0.23 & 0.073 \\
SPA (degree) & 0.31 & $0.016^{\star}$ \\
AoP (degree) & 0.01 & 0.956 \\
WSP (mm) & 0.20 & 0.123 \\
\hline
\end{tabular}

Data are presented as the mean \pm standard deviation (SD). $\mathrm{PD}$, progression distance; AoP, angle of progression; SPA, subpubic arch angle, WSP, width of symphysis pubis. $\mathrm{P}<0.05$ is statistics significance. *, statistical significance.

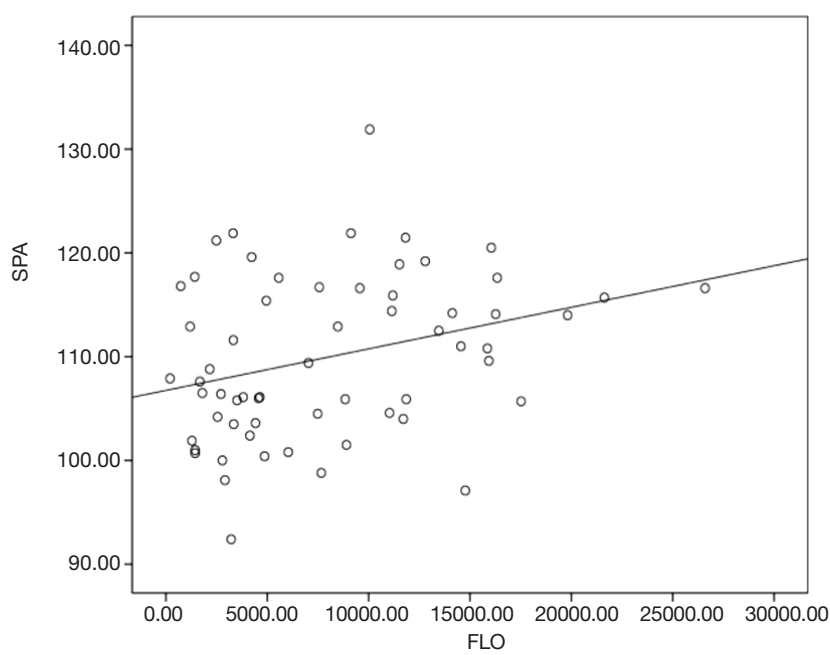

Figure 7 The scatter diagram of the association between all included pregnancies' ultrasound measurements and labor onset, according to these values, the time of labor onset computed by using these two variables was equal to $-298.78+3.937 \times$ SPA. SPA, subpubic arch angle.

Table 5 For 40+ weeks pregnancies the association with labor onset

\begin{tabular}{lcc}
\hline Variable & Pearson correlation coefficient & $P$ \\
\hline PD (mm) & 0.35 & 0.105 \\
SPA (degree) & 0.13 & 0.563 \\
AoP (degree) & 0.09 & 0.697 \\
WSP (mm) & -0.15 & 0.492 \\
\hline
\end{tabular}

Data are presented as the mean \pm standard deviation (SD). PD, progression distance; AoP, angle of progression; SPA, subpubic arch angle, WSP, width of symphysis pubis. $\mathrm{P}<0.05$ is statistics significance.
Table 4 For 39-40 weeks pregnancies the association with labor onset

\begin{tabular}{lcc}
\hline Variable & Pearson correlation coefficient & $\mathrm{P}$ \\
\hline PD $(\mathrm{mm})$ & 0.19 & 0.264 \\
SPA (degree) & 0.33 & 0.050 \\
AoP (degree) & 0.04 & 0.807 \\
WSP (mm) & 0.33 & $0.040^{\star}$ \\
\hline
\end{tabular}

Data are presented as the mean \pm standard deviation (SD). PD, progression distance; AoP, angle of progression; SPA, subpubic arch angle, WSP, width of symphysis pubis. $P<0.05$ is statistics significance. *, statistical significance.

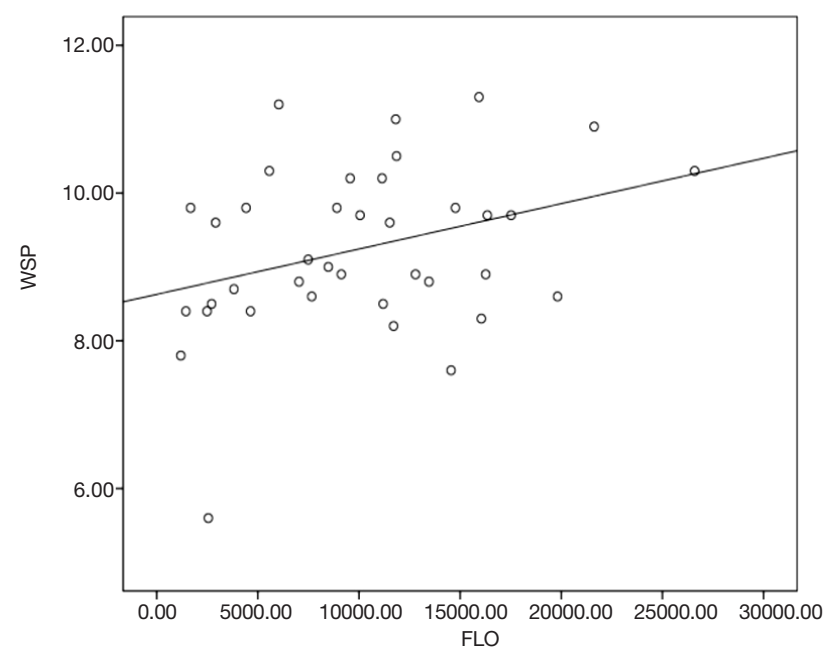

Figure 8 The scatter diagram of the association between 39-40 weeks pregnancies' ultrasound measurements and labor onset. The time of labor onset computed by using these two variables was equal to $-112.12+30.275 \times$ WSP. WSP, width of symphysis pubis.

anterior compartment of the birth canal. These changes may explain the "obstetrical dilemma" hypothesis: that a large-brained/large-bodied fetus requires a wide pelvis, while efficient bipedal locomotion needs a narrow pelvis. The size of the fetal head in the late gestation is only a few centimeters smaller than the widest section of bone canal. The change of pelvis during the pregnancy period could help the fetus passing through the birth canal $(2,17)$. However, our studies found that the association was weaker after 40 weeks than before 40 weeks, because the birth canal was ready for delivery. If no labor occurred, cephalopelvic disproportion may occur. This speculation may need further 
studies to prove.

We observed that SPA gradually increased before delivery, and WSP was much wider when the women were due to delivery before 40 weeks gestation. The results of our study reflect the predictive role of SPA and WSP, which were maintained even after including maternal and antepartum confounding factors. However, we did not find the association between labor onset and the PD and AoP.

However, in this study, there was no association between labor onset and the values of PD and AoP. This result is different from the previous research on the sonographic researches in labor. Before engagement, the fetal head was not fixed; the fetus must twist in a movement known as fetal rotation, finding a suitable way to pass through the birth canal. During the fetal descent towards the outlet, the presenting part undergoes movements of flexion and rotation to replace its largest diameters with the smallest ones (18). Because of the top of the sacrum protruding into the birth canal, the larger back of fetal head fits best against the anterior portion of the birth canal. When the head is in the occiput posterior position, the fetal head may rotate firstly to the occiput anterior position. So, the descending of the fetal head in the occiput posterior position is different from that of fetuses in the occiput anterior position. In the occiput posterior position, the AOP will increase during head descent; however, PD will remain high until flexion starts (19). Therefore, PD and AoP maybe not a suitable predictor of labor onset for the late gestation.

To our knowledge, this study is the first to use SPA, WSP, PD, and AoP to predict the labor onset. Our study provided data about the use of a simple and reliable index of the birth canal and fetal head station in the late gestation. There were several limitations to our study. First, our study is limited by the small number of cases; therefore, further research with large data volume will be necessary to ensure the accuracy of these results. Besides, we should divide the included pregnancies into two subgroups base on the engagement, for further research to clarify the relationship between the labor onset and PD, AoP.

In summary, the use of transperineal ultrasound in the late gestation to predict labor onset is feasible. SPA and WSP are a possible predictive role for labor onset, which can improve the diagnostic accuracy of labor onset. Accurate diagnosis of labor onset could reduce the waste of resource utilization. And in further research, we will try to acquire the cutoff of SPA and WSP for labor onset.

\section{Acknowledgments}

Funding: We gratefully acknowledge the support of the Open Fund of Tianjin Central Hospital of Gynecology Obstetrics/Tianjin Key Laboratory of human development and reproductive regulation (2019XHZ10).

\section{Footnote}

Conflicts of Interest: The authors have no conflicts of interest to declare.

Ethical Statement: The authors are accountable for all aspects of the work in ensuring that questions related to the accuracy or integrity of any part of the work are appropriately investigated and resolved. The local ethics committee approved the study protocol (No. 2019KY038). Eligible patients received oral and written information and provided signed consent forms.

\section{References}

1. Buchmann EJ, Libhaber E. Accuracy of cervical assessment in the active phase of labour. BJOG 2007;114:833-7.

2. Brubaker L, Wolfe AJ. The female urinary microbiota, urinary health and common urinary disorders. Ann Transl Med 2017;5:34.

3. Eggebo TM, Gjessing LK, Heien C, et al. Prediction of labor and delivery by transperineal ultrasound in pregnancies with prelabor rupture of membranes at term. Ultrasound Obstet Gynecol 2006;27:387-91.

4. Ghi T, Youssef A, Martelli F, et al. Narrow subpubic arch angle is associated with higher risk of persistent occiput posterior position at delivery. Ultrasound Obstet Gynecol 2016;48:511-5.

5. Youssef A, Ghi T, Awad EE, et al. Ultrasound in labor: a caregiver's perspective. Ultrasound Obstet Gynecol 2013;41:469-70.

6. Barbera AF, Pombar X, Perugino G, et al. A new method to assess fetal head descent in labor with transperineal ultrasound. Ultrasound Obstet Gynecol 2009;33:313-9.

7. Khorashadi L, Petscavage JM, Richardson ML. Postpartum symphysis pubis diastasis. Radiol Case Rep 2015;6:542.

8. Ghi T, Contro E, Farina A, et al. Three-dimensional ultrasound in monitoring progression of labor: a 
reproducibility study. Ultrasound Obstet Gynecol 2010;36:500-6.

9. Kalache KD, Duckelmann AM, Michaelis SA, et al. Transperineal ultrasound imaging in prolonged second stage of labor with occipitoanterior presenting fetuses: how well does the 'angle of progression' predict the mode of delivery? Ultrasound Obstet Gynecol 2009;33:326-30.

10. Taylor RN, Sonson RD. Separation of the pubic symphysis. An underrecognized peripartum complication. J Reprod Med 1986;31:203-6.

11. Rundell K, Panchal B. Preterm Labor: Prevention and Management. Am Fam Physician 2017;95:366-72.

12. Gold S. The relationship between length of menstrual cycle and date of delivery (predicting the date of confinement). Can Med Assoc J 1957;76:29-34.

13. Albrich SB, Shek K, Krahn U, et al. Measurement of subpubic arch angle by three-dimensional transperineal ultrasound and impact on vaginal delivery. Ultrasound Obstet Gynecol 2015;46:496-500.

14. Haque M, Faruqi NA, Yunus SM. Morphometric Study

Cite this article as: Peng F, Yu Y, Sun Y, Jiang S, Han Y, Zhang $Z$. Using transperineal ultrasound to predict labor onset. Ann Transl Med 2019;7(23):718. doi: 10.21037/atm.2019.12.11 of Subpubic Angle in Human Fetuses. J Clin Diagn Res 2016;10:AC01-4.

15. Aydin S, Bakar RZ, Aydin CA, et al. Assessment of postpartum symphysis pubis distention with $3 \mathrm{D}$ ultrasonography: a novel method. Clin Imaging 2016;40:185-90.

16. Aldabe D, Ribeiro DC, Milosavljevic S, et al. Pregnancyrelated pelvic girdle pain and its relationship with relaxin levels during pregnancy: a systematic review. Eur Spine J 2012;21:1769-76.

17. Trevathan W. Primate pelvic anatomy and implications for birth. Philos Trans R Soc Lond B Biol Sci 2015;370:20140065.

18. Bamberg C, Rademacher G, Guttler F, et al. Human birth observed in real-time open magnetic resonance imaging. Am J Obstet Gynecol 2012;206:505.e1-6.

19. Tutschek B, Torkildsen EA, Eggebo TM. Comparison between ultrasound parameters and clinical examination to assess fetal head station in labor. Ultrasound Obstet Gynecol 2013;41:425-9. 